\title{
Sensing and decision-making components of the signal-regularity effect in vigilance performance
}

\author{
JOEL S. WARM, WILLIAM N. DEMBER, and ANNE Z. MURPHY \\ University of Cincinnati, Cincinnati, Ohio \\ and \\ MARY LYNNE DITTMAR \\ University of Alabama, Huntsville, Alabama
}

\begin{abstract}
A signal-detection-theory analysis was used to assess the sensing and decision-making components of the signal-regularity effect in vigilance performance-enhanced efficiency when critical signals for detection appear in a temporally regular as opposed to a temporally irregular manner. The signal regularity effect was found to be rooted in perceptual sensitivity and was unrelated to response bias. This finding was robust; it occurred with both a simultaneous (comparative judgment) and a successive (absolute judgment) vigilance task. Implications for an expectancy model of the signal-regularity effect are discussed.
\end{abstract}

The signal regularity effect-enhanced performance efficiency when the temporal intervals between critical signals for detection occur in a regular and predictable fashion as opposed to an irregular and unpredictable oneis an old and well-established finding in the study of vigilance or sustained attention (Adams \& Boulter, 1964; Lisper \& Törnros, 1974; Warm, Epps, \& Ferguson, 1974). This effect has primarily been accounted for in terms of an expectancy model proposed by Deese (1955) and modified by Baker $(1959,1963)$. According to that position, subjects in vigilance experiments serve as temporal averaging instruments, forming expectancies as to the approximate schedule of critical signal appearances on the basis of task-related experience. Readiness to detect a signal is assumed to be positively related to the level of expectancy, which is likely to be more veridical when the time course of signal occurrences is predictable than when it is not.

The signal regularity effect appears to be well understood. However, as Loeb and Alluisi (1984) have pointed out, the expectancy model is incomplete in that it does not specify how expectancies affect subjects' readiness to detect signals. Expectancies might somehow "tune" the perceptual system so that it is more responsive to stimulation. It is also conceivable that heightened expec-

Preparation of this manuscript was supported by NASA Grant NAG1-1118 to P. A. Hancock, J. S. Warm, and W. N. Dember, Principal Investigators. The views expressed here are those of the authors and do not necessarily represent those of the named agency. Sandra Hart of NASA Ames Research Center, Moffett Field, CA, was the technical monitor for the grant. We thank Robert Lysaght and Leslie Tudor for help with this project. Correspondence regarding this manuscript should be addressed to Joel S. Warm, Department of Psychology, University of Cincinnati, Cincinnati, OH 45221-0376. tations about when signals are about to appear render subjects more willing to emit detection responses, a result reflecting response bias rather than perceptual sensitivity per se. The issue is reminiscent of the problem of perceptual set and whether its effects reside in the perceptual system itself (perceptual tuning) or in subjects' responses to perceptual experience (Haber, 1966).

This issue can be attacked experimentally by employing a signal-detection-theory analysis (Green \& Swets, 1974) to assess the sensing and decision-making components of the signal-regularity effect and the temporal expectancies presumed to underlie it. Although signaldetection-theory methodology has been widely employed in vigilance research (Davies \& Parasuraman, 1982; Warm \& Jerison, 1984), surprisingly little effort has been made to use it in regard to signal regularity. Except for the passing mention by Parasuraman (1973) of heightened perceptual sensitivity $\left(d^{\prime}\right)$ in the presence of temporally regular signal presentations, the issue remains virtually unexplored. Hence, the present study was designed to determine more fully whether perceptual or response bias factors were at the root of the signal regularity effect. Toward that end, the study utilized two types of tasks featured in a taxonomy of vigilance developed by Davies and Parasuraman (1982). Included were a simultaneous or comparative judgment task, in which all of the information needed to separate signals and nonsignals is present in the stimulus display, and a successive or absolute judgment task, in which subjects are required to appeal to working memory in order to separate signals and nonsignals. Considerable evidence has accumulated to support the meaningfulness of Davies and Parasuraman's task distinction by showing that the effects of several psychophysical factors in vigilance are task dependent (Parasuraman, Warm, \& Dember, 1987; Warm, in press). Accord- 
ingly, these two types of tasks were utilized with an eye toward maximizing the generality of the results to be obtained.

\section{METHOD}

\section{Subjects}

Sixty-four students from the University of Cincinnati, 35 women and 29 men, served as subjects. They ranged in age from 17 to 23 years, with a mean of 20.6. All of the students had normal or corrected-tonormal vision, and all participated in order to fulfill a course requirement.

\section{Apparatus and Procedure}

Sixteen subjects were assigned at random to one of four factorial combinations of task type and signal regularity (simultaneous/regular, simultaneous/irregular, successive/regular, successive/irregular).

All subjects participated in a 60 -min vigil. In all conditions, they monitored the repetitive presentation of a pair of $35 \times 1 \mathrm{~mm}$ vertical red lines (lateral separation $=27 \mathrm{~mm}$ ) which were centered on a black background on a video display terminal. The lines were flashed for a duration of $150 \mathrm{msec}$ once every $2 \mathrm{sec}$, for an event rate of $30 / \mathrm{min}$. In the simultaneous task, critical signals for detection were occasional 2-mm increments in the height of one of the lines in a pair. The spatial position of the higher line (left or right) was varied at random for each subject across the watch. In the successive task, critical signals were occasional 4-mm increments in the height of both lines in a pair. Pilot work insured that the tasks were psychophysically equated at the outset of the watch. The luminance of the stimuli was $.11 \mathrm{~cd} / \mathrm{m}^{2}$ in all experimental conditions.

The vigil was divided into six continuous 10 -min periods of watch. Ten critical signals were presented per period in all conditions (signal probability $=.033$ ). In the regular signal conditions, critical signals appeared once every $60 \mathrm{sec}$. In the irregular signal conditions, critical signals appeared on the average of once per minute, but with intersignal intervals (ISIs) of $24,48,72,96$, and $120 \mathrm{sec}$. These intervals were varied at random for each subject within each 10-min period, with the restriction that critical signals were separated three times by ISIs of 24 and $72 \mathrm{sec}$, twice by an ISI of $48 \mathrm{sec}$, and once each by ISIs of 96 and $120 \mathrm{sec}$.

Subjects were tested individually in an Industrial Acoustics sound chamber. They were seated in front of a table containing the video display terminal, which was mounted at eye level. Ambient illumination in the chamber was provided by a 15 -W bulb shielded by a conical light fixture. The fixture was positioned away from the display in order to minimize glare. An Apple Ile microcomputer generated the stimuli and controlled the presentation of critical signals and neutral events in all experimental conditions. The computer also recorded the subjects' responses. The subjects indicated their detection of critical signals by depressing the spacebar on the computer keyboard. In all conditions, responses occurring within $1.85 \mathrm{sec}$ after the onset of a critical signal were recorded automatically as correct detections (hits). All other responses were recorded as errors of commission (false alarms). Subjects surrendered their watches at the outset of the vigil and had no knowledge of its duration other than that it would not exceed $90 \mathrm{~min}$.

\section{RESULTS}

\section{Traditional Measures}

Percentages of correct detections and false alarms were determined for each subject within each period of watch. Mean percentages of correct detections in all experimental conditions are presented in Table 1 .

An analysis of variance based upon an arcsin transformation of the data of Table 1 indicated that the probability of signal detection was significantly greater when critical signals appeared in a temporally regular $(M=66.5 \%)$ as opposed to an irregular $(M=56 \%)$ fashion $[F(1,60)=$
Table 1

Mean Percentages of Correct Detections in All Experimental Conditions

\begin{tabular}{llccccccc}
\hline & & \multicolumn{7}{c}{ Periods of Watch (10 min) } \\
\cline { 3 - 8 } \multicolumn{1}{c}{ Task } & Regularity & 1 & 2 & 3 & 4 & 5 & 6 & $M$ \\
\hline Simultaneous & Regular & 85 & 74 & 60 & 65 & 61 & 56 & 67 \\
& Irregular & 76 & 63 & 59 & 51 & 49 & 38 & 56 \\
Successive & Regular & 80 & 69 & 65 & 63 & 61 & 59 & 66 \\
& Irregular & 71 & 64 & 54 & 56 & 45 & 43 & 56 \\
$M$ & & 78 & 68 & 60 & 59 & 54 & 49 & \\
\hline
\end{tabular}

$5.22, p<.05]$, and that performance efficiency declined significantly over the course of the watch $[F(5,300)=$ $33.43, p<.001]$. No significant difference in the overall probability of signal detection was noted between the two types of vigilance tasks ( $M$ s for the simultaneous and successive tasks were $62 \%$ and $61 \%$, respectively, $F<1$ ), and all of the interactions in the analysis lacked significance $(p>.05)$.

A similar analysis based upon an arcsin transformation of the percentages of false alarms indicated that the frequency of false alarms declined significantly with time on task [Ms for Periods 1-6 $=6.6 \%, 5.2 \%, 5.5 \%, 5.1 \%$, $4.2 \%$, and $4.0 \%$, respectively; $F(5,300)=9.79, p<$ $.001]$, and that the overall likelihood of false alarms was significantly greater when subjects were confronted with the successive $(M=6.8 \%)$ than when they were confronted with the simultaneous $(M=2.8 \%)$ task $[F(1,60)=$ $12.36, p<.001]$. None of the remaining sources of variance in the analysis of the false-alarm data were significant $(p>.05)$.

\section{Signal-Detection-Theory Measures}

Nonparametric indices of perceptual sensitivity $\left(A^{\prime}\right)$ and response bias $\left(B^{\prime \prime} ;\right.$ Grier, 1971) were computed from the percentages of correct detections and false alarms for each subject in all experimental conditions. Nonparametric measures were used in response to arguments that the assumptions governing the use of their parametric analogs $d^{\prime}$ and $\beta$ (normality and equal variance in the noise and signal-plus-noise distributions) are unlikely to be met in vigilance experiments (Warm \& Jerison, 1984).

Mean $A^{\prime}$ scores for the regular and irregular signal conditions are plotted as a function of time on watch in Figure 1. Data for the simultaneous and successive tasks are presented separately in each panel.

It is evident in the figure that perceptual sensitivity was greater with regular than with irregular signal presentations, and that this effect was obtained with both the simultaneous and successive tasks. Also evident in the figure is a decline in perceptual sensitivity across the watch, a result observable with both types of tasks and in both conditions of signal regularity. An analysis of variance of the $A^{\prime}$ scores revealed significant main effects for signal regularity $[F(1,60)=5.45, p<.025]$ and for periods of watch $[F(5,300)=15.42, p<.001]$. In addition, perceptual sensitivity was significantly greater in the simul- 


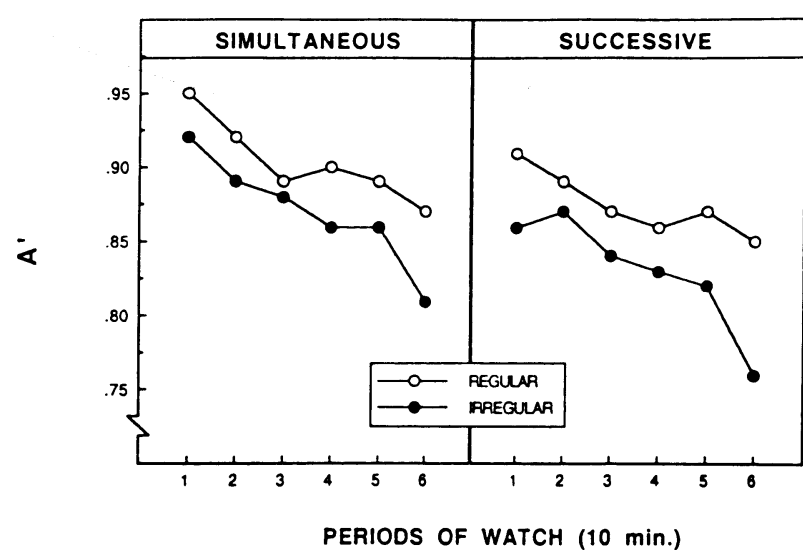

Figure 1. Perceptual sensitivity for regular and irregular signals as a function of periods of watch. Data for the simultaneous and successive tasks are plotted separately in each panel. ( $A^{\prime}$ scores can range from .50 , chance performance, to 1.00 , maximum sensitivity.)

taneous $(M=.89)$ than in the successive $(M=.86)$ task $[F(1,60)=4.25, p<.05]$. There were no significant interactions in the analysis $(p>.05)$.

An analysis of variance of the data for the response criterion measure $\left(B^{\prime \prime}\right.$, which can vary from -1 , extreme leniency, to +1 , extreme conservatism) indicated that subjects became more conservative in responding across the watch $[M s$ for Periods $1-6=.22, .42, .44, .51, .61, .52$, respectively; $F(5,300)=6.90, p<.001]$. No other source of variance in the analysis of the $B^{\prime \prime}$ scores was statistically reliable $(p>.05)$.

\section{DISCUSSION}

A signal-detection-theory analysis was employed in this study to assess the sensing and decision-making components of the signal-regularity effect in vigilance performance. The study was prompted by the observation that the expectancy model, which is commonly used to accoun for this effect, is not specific as to the manner in which heightened expectations in the presence of temporally regular signals aid in the detection of such signals. The results indicated that the probability of signal detection in both the simultaneous and successive formats was greater when critical signals appeared in a temporally regular than in an irregular fashion, and that this outcome was accompanied by parallel changes in perceptual sensitivity. No evidence was obtained for any association between signal regularity and changes in the subjects' response criterion. Thus, the present findings indicate that the signal-regularity effect is broadbased, that it is rooted in perceptual factors, and that it does not simply reflect an increased willingness on the part of subjects to emit detection responses. This result confirms Parasuraman's (1973) earlier report of heightened perceptual sensitivity under conditions of signal regularity. It is also consistent with the results of Egan, Greenberg, and Schulman's (1961) study, which demonstrated that even under alerted conditions, uncertainty about the time of signal presentation degrades perceptual sensitivity.

From the point of view of expectancy theory, it would appear that the beneficial effects of heightened expectations in the presence of signal regularity are also perceptual in origin, and that efforts to further understand the implications of expectancy formation in regard to the signal-regularity effect should treat the issue as a perceptual problem. While the present study was not designed to investigate specific features in the expectancy/perception relation, we can suggest some possible ways in which heightened levels of expectation might increase the perceptibility of signals in visual vigilance tasks, such as those utilized in this investigation.
Visual vigilance tasks are typically considered to be "loosely coupled," since subjects are free to make head and eye movements which are incompatible with viewing the monitored display (Warm \& Jerison, 1984). When subjects anticipate the imminent arrival of a critical signal, they may be more likely to engage in appropriate observing behaviors such as reducing their head movements and orienting their gaze directly to the display. Such behavior would increase the chances of foveal rather than peripheral viewing, thereby enhancing visual acuity and rendering the signals more salient. Moreover, reductions in head movements would reduce the parallax changes which make the separation of signals from noise more difficult with the spatial discriminations required in the present study. Indeed, Warm, Chin, Dittmar, and Dember (1987), who used the same display as the one in the present study, found that close coupling achieved through head stabilization enhanced signal detectability in the case of both simultaneous and successive vigilance tasks.

While observing behaviors that decrease the signal-to-noise-ratio in the presence of heightened levels of expectation may be one route through which expectancy formation affects signal detectability, it is worth noting that subjects miss signals in a vigilance task even when the display is visually fixated (Mackworth, Kaplan, \& Metlay, 1964). Accordingly, more central factors, such as increments in the processing resources devoted to the task (Davies \& Parasuraman, 1982; Wickens, 1991), may also be involved in the role played by expectancy formation in the signalregularity effect.

In addition to its implications for the signal-regularity effect, the results of this study also have ramifications for the decrement functionthe decline in the probability of signal detections across the watch which typifies vigilance performance. In the present case, the decrement in detections was accompanied by criterion elevations and by reductions in perceptual sensitivity in both the simultaneous and successive tasks. In the original formulation of their vigilance taxonomy, Davies and Parasuraman (1982) suggested that criterion-based docrements were common in vigilance experiments, but that sensitivity decrements were restricted to successive tasks in the presence of fast event rates ( $>24 / \mathrm{min}$ ). On the basis of later findings, however, Parasuraman and his colleagues (Parasuraman et al., 1987) concluded that sensitivity decrements could also occur in the case of simultaneous tasks when a fast event rate was combined with difficult discrimination conditions. The simultaneous task criterion decrement in the present study is consistent with the latter view, since this study featured a fast event rate $(30 / \mathrm{min})$ and required subjects to discriminate small (2- or 4-mm) changes in the height of very briefly exposed $(150 \mathrm{msec}$ ) stimuli. Hence, the results of this study provide additional evidence for the occurrence of a sensitivity decrement in a simultaneous vigilance task.

\section{REFERENCES}

Adams, J. A., Boulter, L. R. (1964). Spatial and temporal uncertainty as determinants of vigilance behavior. Journal of Experimental Psychology, 67, 127-131.

BAKER, C. H. (1959). Towards a theory of vigilance. Canadian Journal of Psychology, 13, 35-42.

BAKER, C. H. (1963). Further toward a theory of vigilance. In D. N. Buckner \& J. J. McGrath (Eds.), Vigilance: A symposium (pp. 127170). New York: McGraw-Hill.

Davies, D. R., \& Parasuraman, R. (1982). The psychology of vigilance. London: Academic Press.

DeEse, J. (1955). Some problems in the theory of vigilance. Psychological Review, 62, 359-368.

Egan, J. P., Greenberg, G. Z., \& Schulman, A. I. (1961). Interval of time uncertainty in auditory detection. Journal of the Acoustical Society of America, 33, 771-778.

Green, D. M., SWETs, J. A. (1974). Signal detection theory and psychophysics. Huntington, NY: Krieger.

GrIer, J. B. (1971). Nonparametric indexes for sensitivity and bias: Computing formulas. Psychological Bulletin, 75, 424-429.

Haber, R. N. (1966). Nature of the effect of set on perception. Psychological Review, 73, 335-351.

LISPER, H. O., \& TÖRNROS, J. (1974). Effects of inter-signal regularity on increase in reaction time in a one hour auditory monitoring task. Acta Psychologica, 38, 455-460. 
Loeb, M., \& Alluisi, E. A. (1984). Theories of vigilance. In J. S. Warm (Ed.), Sustained attention in human performance (pp. 179-205). Chichester, U.K.: Wiley.

Mackworth, N. H., Kaplan, I. T., \& Metlay, W. (1964). Eye movements during vigilance. Perceptual \& Motor Skills, 18, 397-402.

Parasuraman, R. (1973). Vigilance and attention: A psychophysiological study. Unpublished master's thesis, University of Aston in Birmingham, Birmingham, U.K.

Parasuraman, R., Warm, J. S., \& Dember, W. N. (1987). Vigilance: Taxonomy and utility. In L. S. Mark, J. S. Warm, \& R. L. Huston (Eds.), Ergonomics and human factors: Recent research (pp. 11-32). New York: Springer-Verlag.

WARM, J. S. (in press). Vigilance and target detection. In B. M. Huey \& C. D. Wickens (Eds.), Teams in transition. Washington, DC: National Academy Press.
Warm, J. S., Chin, K., Dittmar, M. L., \& Dember, W. N. (1987). Effects of head restraint on signal detectability in simultaneous and successive vigilance tasks. Journal of General Psychology, 114, 423-431.

Warm, J. S., EpPS, B. D., \& Ferguson, R. P. (1974). Effects of knowledge of results and signal regularity on vigilance performance. Bulletin of the Psychonomic Society, 4, 272-274.

WARM, J. S., \& JERISON, H. J. (1984). The psychophysics of vigilance. In J. S. Warm (Ed.), Sustained attention in human performance (pp. 15-59). Chichester, U.K.: Wiley.

WiCKens, C. D. (1991). Processing resources and attention. In D. L. Damos (Ed.), Multiple-task performance (pp. 3-34). London: Taylor \& Francis.

(Manuscript received February 19, 1992.)

\section{The Australia Prize}

The Australia Prize is an international award given by the Government of the Commonwealth of Australia for an outstanding specific achievement in a selected area of science and technology promoting human welfare. In this context, science encompasses natural and technological sciences, engineering, and mathematics. A prize is $\$ A 250,000$, together with an inscribed medal.

The Australia Prize Committee consists of the Presidents of the Australian Academy of Science and the Australian Academy of Technological Sciences and Engineering, alternating in the Chair, and other distingished Australans. The Committee, assisted by specialist advisers, recommends on the award of the Australia Prize.

The Australia Prize acknowledges outstanding achievements by scientists and technologists, and celebrates success. To date, the awards have featured innovations based on applying science and technology to human challenges.

\section{THE 1993 AUSTRALIA PRIZE}

The 1993 Australia Prize will be awarded in the field of

\section{SENSORY PERCEPTION}

Nominations are now being sought internationally from learned bodies, universities, research institutions, and professional associations. Nominations close on July 31, 1992.

Copies of the nomination form may be obtained from the Psychonomic Society Publications Office, 1710 Fortview Road, Austin, TX 78704 (Telephone 512-462-2442).

For further information, contact the Australia Prize Secretariat at the following address: Australia Prize, GPO Box 9839, Canberra, ACT 2601, Australia (Telephone 61-6-276-1510, Fax 616-276-1827, Telex AA 62654). 